\title{
Editorial
}

\section{Tabaco: um problema pediátrico}

\author{
Tobacco: a pediatric problem
}

Ana Cecilia P. R. Marques ${ }^{1}$, Ronaldo Ramos Laranjeira ${ }^{2}$

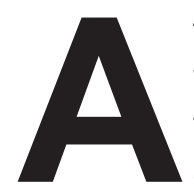

família é a matriz para a formação e desenvolvimento dos indivíduos que a compõem, transmitindo valores, regras, costumes e ideais. Sua influência vai desde padrões de comportamento até a qualidade da saúde de seus membros em todas as etapas da vida. Se o modelo parental tem como característica o uso do tabaco, os demais membros da família, principalmente as crianças, sofrerão esse impacto de forma direta - achados comprovados por pesquisas internacionais e nacionais ${ }^{(1,2)}$.

As repercussões do uso de drogas psicotrópicas por gestantes tem sido alvo de estudos na atualidade. Além de atravessarem a placenta e modificarem o desenvolvimento do feto, a manutenção do uso da substância pela mãe após o parto pode acarretar diferentes problemas por meio do aleitamento e do ambiente poluído ${ }^{(3,4)}$. O tabaco diminui a produção de leite, a concentração de gordura do leite e reduz o tempo de amamentação, além de agredir drasticamente as vias aéreas da lactante e das crianças ${ }^{(5)}$. A nicotina se acumula no leite humano cerca de 30 minutos após o consumo de um cigarro e pode ser encontrada em concentrações até três vezes maiores que no sangue materno(5-9). A exposição à fumaça aumenta o risco de várias doenças como otite, bronquite, pneumonia, irritação do trato respiratório superior, redução da função pulmonar, aumento do número de episódios e da gravidade de cri-

\section{Referências bibliográficas}

1. Pratta EM, Santos MA. Opiniões dos adolescentes do ensino médio sobre o relacionamento familiar e seus planos para o futuro. Paideia 2007;17: 103-14.

2. Ramirez RM, Andrade D. La familia y los factores de riesgo relacionados con el consumo de alcohol y tabaco en los niños y adolescentes. Rev Lat-Am Enfermagem 2005;13:813-8.

3. Del Ciampo LA, Ricco RG, Almeida CA. Aleitamento materno: passagens e transferências mãe-filho. São Paulo: Atheneu; 2004. ses de asma(10,11). Neste número da Revista Paulista de Pediatria, Del Ciampo et $a^{(12)}$ encontraram resultados alarmantes em pesquisa com gestantes: 19,2\% eram tabagistas ativas, $28,2 \%$ tabagistas passivas e $16,8 \%$ tabagistas ativas e passivas, o que mostra uma prevalência acima da população geral feminina.

E o problema não acaba aí. A adolescência, uma etapa de desenvolvimento biológico e psicossocial do indivíduo de grande vulnerabilidade, é outro momento no qual a influência da família é fundamental. Moreno et a/ ${ }^{(13)}$ estudaram uma amostra de estudantes adolescentes de escolas municipais, cujo principal achado foi o fato de que ser adolescente usuário de bebida alcoólica ou de tabaco está associado significativamente com ter familiar que bebe e fuma. A influência do meio familiar é contínua e fator preponderante no equilíbrio do indivíduo; além do mais, o início precoce de tabaco aumenta muito as chances do desenvolvimento da dependência e de agravos à saúde na idade adulta ${ }^{(14,15)}$.

A família, dessa maneira, exerce um papel primordial na construção e formação do indivíduo, mas uma avaliação pediátrica aprofundada no tema pode redimensionar esse impacto. Ações de prevenção e promoção à saúde devem ser ampliadas junto às escolas, assim como ações educativas sistemáticas e continuadas precisam ser repetidas nos programas de assistência pré-natal e de puericultura.
4. Parackal S, Ferguson E, Harraway J. Alcohol and tobacco consumption among 6-24-months post-partum New Zealand womem. Matern Child Nutr 2007;3:40-51.

5. Dahlström A, Ebersjö C, Lundell B. Nicotine exposure in breastfed infants. Acta Paediatr 2004;93:810-6.

6. Laurberg P, Nohr SB, Pedersen KM, Fuglsang E. lodine nutrition in breastfed infants in impaired by maternal smoking. J Clin Endocrinol Metab 2004;89:181-7. 
7. Knudsen A. Tobacco - enemy of public health from conception to grave. Acta Paediatr 2004;93:1420.

8. Stepans MB, Wilhelm SL, Dolence K. Smoking hygiene: reducing infant exposure to tobacco. Biol Res Nurs 2006;8:104-14.

9. Mascola MA, van Vunakis H, Tager IB, Speizer FE, Hanrahan JP. Exposure of young infants to environmental tobacco smoke: breast-feeding among smoking mothers. Am J Public Health 1998;88:893-6.

10. Mello PR, Pinto GR, Botelho $C$. The influence of smoking on fertility, pregnancy and lactation. J Pediatr (Rio J) 2001;77:257-64.

11. Del Ciampo LA, Almeida CA, Ricco RG. The children as a passive smoking. Rev Paul Ped 1999;17:74-8.
12. Del Ciampo LA, Ricco RG, Ferraz IS, Daneluzzi JC, Martinelli Jr CE. Prevalência de tabagismo e consumo de bebida alcoólica em mães de lactentes menores de seis meses de idade. Rev Paul Pediatr 2009;27:361-5.

13. Moreno RS, Ventura RN, Bretãs JR. Ambiente familiar e consumo de álcool e tabaco entre adolescentes. Rev Paul Pediatr 2009;27:354-60.

14. Baus J, Kupek E, Pires M. Prevalência e fatores de risco relacionados ao uso de drogas entre escolares. Rev Saude Publica 2002;36: 40-6.

15. Ramirez RM, Andrade D. La familia y los factores de riesgo relacionados con el consumo de alcohol y tabaco en los niños y adolescentes. Rev Lat-Am Enfermagem 2005;13:813-8.
Instituição: Universidade Federal de São Paulo (Unifesp), São Paulo, SP, Brasil

'Doutora em Ciências pela Unifesp, São Paulo, SP, Brasil

2Professor titular do Departamento de Psiquiatria da Unifesp, São Paulo, SP, Brasil
Endereço para correspondência: Ana Cecilia P. R. Marques

Rua Oscar Freire, 530, conjunto 52 CEP 01426-001 - São Paulo/SP

E-mail: rmarq@terra.com.br 